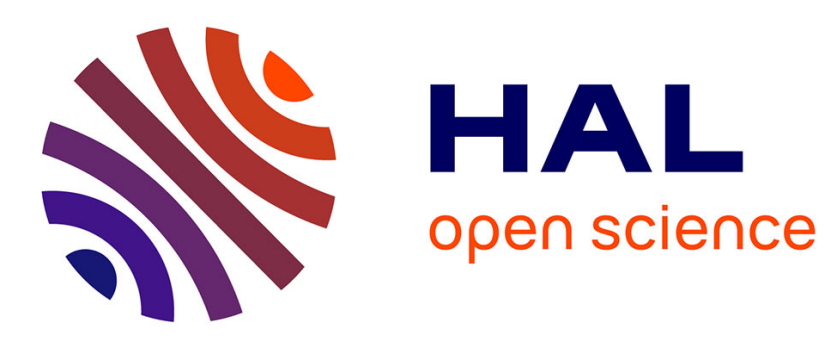

\title{
Malaise dans les start-up. Entre désir héroïque et anxiété créatrice
}

Romain Buquet, Nathalie Luca, Jean-Philippe Bouilloud

\section{To cite this version:}

Romain Buquet, Nathalie Luca, Jean-Philippe Bouilloud. Malaise dans les start-up. Entre désir héroïque et anxiété créatrice. Nouvelle revue de psychosociologie, 2017, 24 (2), pp.93-111. 10.3917/nrp.024.0093 . halshs-01821670

\section{HAL Id: halshs-01821670 \\ https://shs.hal.science/halshs-01821670}

Submitted on 23 Jul 2019

HAL is a multi-disciplinary open access archive for the deposit and dissemination of scientific research documents, whether they are published or not. The documents may come from teaching and research institutions in France or abroad, or from public or private research centers.
L'archive ouverte pluridisciplinaire HAL, est destinée au dépôt et à la diffusion de documents scientifiques de niveau recherche, publiés ou non, émanant des établissements d'enseignement et de recherche français ou étrangers, des laboratoires publics ou privés. 


\title{
Malaise dans les start-up Entre désir héroïque et anxiété créatrice
}

\author{
Romain Buquet, Nathalie Luca et Jean-Philippe Bouilloud
}

Cet article porte sur l'anxiété des entrepreneurs du numérique ou startuppers ${ }^{1}$. Figures médiatisées de la puissance et du succès (Mark Zuckerberg pour Facebook, Jeff Bezos pour Amazon...), les start-uppers s'affichent en " thaumaturges " des temps modernes et offriraient une nouvelle image du héros. Cette image est renforcée par les médias et les politiques, séduits par l'intrusion disruptive de nouveaux modèles économiques et organisationnels qui bouleversent le jeu économique, suscitent des vocations chez les jeunes cadres et obligent les entreprises établies à repenser le schéma de leurs activités. Ce nouveau modèle entrepreneurial connaît un engouement sans précédent de la part de certaines catégories de " jeunes professionnels " et en même temps suscite de nombreuses critiques par le coût social que ces ruptures induisent ou la dégradation des conditions de travail qu'elles entraînent. Pour de nombreux jeunes diplômés, ce modèle est devenu en quelques années la voie privilégiée pour un nombre croissant d'entre eux et attire simultanément une part significative des salariés en reconversion. Ainsi, par exemple, plus de $40 \%$ de la promotion du mba de Stanford (Moules, 2015) et un quart de la

Romain Buquet, doctorant, escp Europe. romain.buquet@edu.escpeurope.eu Nathalie Luca, directrice du CéSor (Centre d'études en sciences sociales du religieux), cnrs/ehess.nathalie.luca@ehess.fr

Jean-Philippe Bouilloud, professeur, escp Europe.bouilloud@escpeurope.eu

${ }^{1}$ Le terme correct en français pour " start-up » est " jeune pousse », on devrait donc dire " entrepreneur de jeune pousse », ce qui est peu employé 
promotion 2013 de hec (contre $10 \%$ des diplômés dix ans auparavant $)^{2}$ ont créé leur entreprise en dépit des formidables opportunités de carrière qu'ils pouvaient obtenir par ailleurs.

La littérature et l'image médiatique dressent le plus souvent un portrait élogieux de ces héros modernes. On voit cependant apparaître des témoi-gnages révélant les écarts entre l'idéal présenté et le vécu de ceux qui y travaillent. On peut considérer que par certains côtés les start-uppers s'inscrivent dans un courant critique du capitalisme qu'Ève Chiapello fait remonter au milieu du xix ${ }^{e}$ siècle. II s'agit de la " critique artiste ", portée par des artistes, des écrivains, des étudiants qui « rêvent d'un mode de vie délivré de toute oppression et célèbrent les vertus de l'imagination et la créativité », critiquent le " désenchantement du monde » et " placent la liberté au-dessus de tout». Ainsi d'ailleurs les start-uppers interviewés se considèrent-ils bien plus comme des " artistes ", des créateurs que comme des chefs d'entreprise. La critique artiste " accompagna la transfor- mation du travail vers plus de flexibilité et de mobilité depuis les années 1970. Son antiautoritarisme alimenta les nouveaux modes de gestion fondés sur une réduction du nombre de niveaux hiérarchiques, le déve-loppement de l'autonomie au travail, l'éclatement des grandes techno-structures en flottilles d'entreprises beaucoup plus malléables »(Chiapello, 1998, 2004), au sein desquelles apparaîtront plus tard les start-up.

II apparaît nécessaire d'appréhender clairement les enjeux psycho-sociaux de ce qui se présente pour certains comme une révolution. Dans cette perspective, nous avons conduit dix-neuf entretiens cliniques de type "récit de vie " dans un incubateur $^{3}$ phare de start-up parisiennes : le numa (NUmérique + huMAin), connu depuis sa création en 2000 comme

"Silicon Sentier ». Les résultats présentés ici n'ont pas volonté à être généralisés et demanderaient d'être approfondis par d'autres études com- plémentaires. En l'état, ils montrent néanmoins que ces jeunes entre-preneurs présentent des profils d'instabilité émotionnelle et d'anxiété récurrente qui ne réduisent en rien leur engagement et volonté de changer le monde : bien au contraire, l'anxiété transversale qui émane de leur propos est indissociable de leur création entrepreneuriale.

Précisons d'emblée que la notion même d'« entrepreneur " ne fait pas consensus parmi les chercheurs et nous ne disposons pas d'une définition unique et reconnue par tous. Dans cet article, nous utilisons le concept d'entrepreneur tel que défini par Joseph A. Schumpeter (1934) : celui ou celle qui casse l'équilibre des marchés et fait avancer le déve-loppement économique par un processus de " destruction créatrice ». Les entrepreneurs du web (ou start-uppers), qui sont l'objet de notre

\footnotetext{
${ }^{2}$ Premier baromètre Entrepreneuriat hec, 2015, https://fr.slideshare.net/HECParis/ 1erbarometre-entrepreneuriat-hec.

${ }^{3}$ Incubateur : structure d'accompagnement de projets de création d'entreprises
} 
recherche,- correspondent bien à cette définition. Ils forment une souscatégorie, tout en constituant des cas extrêmes.

Dans une première partie, nous dressons un état de l'art des quelques travaux sur l'anxiété et la stabilité émotionnelle des entrepreneurs. Parmi eux, aucun ne semble s'être concentré sur la spécificité des start-uppers. C'est ce que nous tâchons modestement de défricher dans la deuxième partie, à partir des données recueillies auprès de dix-neuf entrepreneurs du numérique. Le nombre réduit de participants et leur spécificité nous interdisent toute tentative de généralisation, mais nous offrent une opportunité d'exploration. Enfin, nous concluons sur le paradoxe émotionnel qui semble habiter le start-upper.

\section{Anxiétés et entrepreneurs}

Si on entend par anxiété les " manifestations d'inquiétude et de préoc-cupation ${ }^{4}$ " des individus face aux événements sociaux qui les environnent, alors le monde du travail génère structurellement de l'anxiété dans la période contemporaine : emplois non sécurisés (Linhart, Rist et Durand, 2002), perte de sens du travail (Hanique, 2014), situations structurelles d'in-jonctions paradoxales (Bouilloud, 2012 ; Pérezts, Bouilloud et Gaulejac, 2011), voire généralisation de l'idéal comme norme au travail (Dujarier,

2006) sont quelques-unes des raisons de cette anxiété. Cela débouche sur un éventail de problèmes de santé et de santé mentale (Clot et Lhuilier, 2010), regroupés sous l'appellation trop large mais convenue des « risques psychosociaux».

Si la notion d'angoisse a bien été étudiée en psychanalyse, en tant que " détresse psychique " et " réaction du moi face au danger " ayant des impacts physiologiques (Freud, 1975), la notion d'anxiété demeure à travailler en sciences sociales. Elle est absente des vocabulaires de psychosociologie (Barus-Michel, Enriquez et Lévy, 2002), de psychanalyse (Laplanche et Pontalis, 1968) ou de sociologie (Boudon et Bourricaud, 1990). Elle n'apparaît que dans une très brève notice de l'Encyclopédie philosophique universelle dans laquelle anxiété et angoisse sont associées, la seconde se différenciant par ses manifestations organiques (Auroux, 1990). Le Trésor de la langue française (tIf) du cnrs définit l'anxiété comme un «trouble psychique accompagné de phénomènes physiques »

- proche en ce sens de l'angoisse, ou comme "l'appréhension d'une menace intérieure souvent indéfinissable ». Venant du latin « anxietas » qui signifie "disposition naturelle à l'inquiétude », elle se traduit par un état général tendu et peut se manifester par des moments de crise. Ainsi, à la différence de l'angoisse, l'anxiété correspond davantage à un senti-ment diffus qui se déploie sur le temps long, quand l'angoisse relève d'un

${ }^{4}$ Centre national de ressources textuelles et lexicales/Inquiétude 
moment critique où le danger est perceptible et conduit à une réaction physiologique manifeste et immédiate.

Comme on le verra, ce que l'on observe chez les start-uppers interviewés relève de cette définition de l'anxiété. Elle se manifeste par leur compor-tement général mis en alerte par un environnement très incertain. On notera aussi que des moments-clés de la vie de leur entreprise (présenta-tion à des investisseurs, évaluation par des fonds, etc.) génèrent ce pic d'inquiétude et de manifestations physiologiques associé à l'angoisse. Mais il y a un autre sens de l'anxiété que nous signale le tlf, celui de " désir intense, extrême tension nerveuse d'une attente vécue, non plus dans la crainte, mais dans le plaisir ". Cette définition n'est pas seule-ment centrée de façon négative sur la crainte et la peur (qu'Aristote définissait comme une " attente du malheur », Eth. Nic., III, VI, 2), mais aussi de façon positive sur une espérance de succès. C'est cette anxiété, biface, à la fois d'inquiétude et d'espoir, que nous avons trouvée chez les start-uppers du numa. C'est cette double dimension de l'anxiété, à la fois comme inquiétude mais aussi comme tension positive vers la réalisation d'un projet révolutionnaire, que nous voulons mettre en avant dans la notion d'“ anxiété créatrice »: c'est une anxiété qui reflète cette dispo-sition à l'inquiétude de l'anxietas, et en même temps elle est créatrice au sens de la « destruction créatrice » de l'entrepreneur schumpéterien qui détruit des modes anciens de production et les remplace par ceux qu'il propose (cas d'Uber ou Amazon par rapport à leurs secteurs respectifs).

\section{L'entrepreneur, un héros moderne?}

La littérature gestionnaire sur les start-up croise de multiples champs de recherche, notamment sur l'entrepreneur, le management des petites entreprises et le leadership. En effet, le créateur de start-up est à la fois un entrepreneur, mais spécifique, un manager, mais dans une organisa-tion singulière, et un dirigeant-actionnaire concerné par les problématiques de leadership. Tous ces termes ne bénéficient pas d'une définition stable ou d'une théorie unifiée et celui d' " entrepreneur », pourtant ancien et bien étudié, est abordé avec des termes de référence différents en économie, sociologie, psychologie, anthropologie ou finance (Low et MacMillan, 1988).

Dans la littérature de gestion, l'entrepreneur est souvent décrit de manière louangeuse. Dès 1985, lors de la parution de la première édition du Journal of Business Venturing, on prête à l'entrepreneur toutes les compétences des dirigeants et plus encore : il tolère les situations ambiguës, agit de manière autonome, résiste à la conformité, fait preuve d'adresse dans ses relations interpersonnelles, s'adapte rapidement au changement et sollicite rarement de l'aide (Sexton et Bowman, 1985), même si les auteurs soulignent déjà que ces atouts hors du commun peuvent générer du stress et de la solitude. Pour la recherche en gestion, l'entrepreneur est habituellement vu comme plus épanoui que la moyenne de ses contemporains (Cross et Travaglione, 2003), plus satisfait de sa vie et de sa carrière que 
les employés (Blanchflower et Oswald, 1998) et, de manière générale, il présenterait une plus grande tolérance au stress (Rauch et Frese, 2007). Cette " stabilité émotionnelle ", ou résistance à l'anxiété, est d'ailleurs associée à un gage de performance (Singh, 1989). De manière analogue, leur confiance dans les opportunités affecterait positivement leur succès entrepreneurial (Dimov, 2010). Face à ceux, cadres ou employés, qui vivent des tensions réelles entre vie privée et vie professionnelle, les entrepreneurs auraient trouvé une harmonie entre aspirations professionnelles, projet personnel et impact social de leur action (Gomez-Breysse, 2016). Cette conviction dans l'intérêt - ou l'avenir - de leur projet mais aussi de leur vision de la société ou du travail expliquerait qu'ils surestiment leurs chances d'avoir raison (Busenitz et Barney, 1997) : ayant foi dans leurs projets, les entrepreneurs présenteraient un niveau d'optimisme supérieur

à la moyenne (Abdelsamad et Kindling, 1978 ; Fraser et Greene, 2006 ; Lowe et Ziedonis, 2006) et ils s'attendraient à des résultats positifs même quand ces attentes ne sont pas rationnelles. Cet optimisme peut donc altérer leur faculté de jugement, être contre-productif pour les résultats de l'entreprise en termes tant de revenus que de création d'emplois (Hmieleski et Baron, 2009) : au final l'excès de confiance serait la première cause de faillite entrepreneuriale (Gartner, 2004).

Cet enthousiasme est plus propre au créateur d'entreprise qu'à celui qui la dirige : les dirigeants qui ont créé leur entreprise seraient plus stables et plus indépendants (self-assertive) que ceux qui ont repris une entre-prise familiale (Brandstätter, 1997). L'entrepreneur n'est pas uniquement un dirigeant d'une organisation : il en est le créateur, il en revendique l'idée, la paternité (on verra plus loin comment la métaphore familiale est omniprésente), il en est à la fois responsable et principal bénéficiaire puisqu'il en est aussi, souvent, le principal actionnaire. L'investissement narcissique est donc majeur, avec ses enjeux positifs de possible, mais rare, réussite grandiose et négatifs d'échec statistiquement très probable. C'est dans cette tension qu'apparaît l'anxiété.

\section{Une anxiété réelle mais peu analysée}

De manière générale, les traits positifs des entrepreneurs ont été plus étudiés que les difficultés psychiques auxquelles ils sont confrontés. Quelques travaux ont évoqué l'anxiété et le déséquilibre émotionnel de l'entrepreneur, notamment au sujet de leur principale préoccupation, la levée de fonds (Blanchflower et Oswald, 1998). Enfin, une étude récente montre que les entrepreneurs sont aussi largement sujets à la dépression, qui toucherait $30 \%$ d'entre eux (Freeman et coll., 2015), contre une prévalence dans la population générale française de $7,5 \%{ }^{5}$. On trouve

\footnotetext{
${ }^{5}$ La prévalence de l'épisode dépressif caractérisé (edc) au cours des douze derniers mois telle que mesurée par le cidi-sf dans le cadre du baromètre Santé de l'inpes s'élève en France, en 2010, à 7,5 \% parmi les 15-85 ans.
} 
cependant encore peu de choses sur cette catégorie particulière d'entre-preneurs que sont les start-uppers. Pourtant, l'investissement narcissique de ces créateurs d'entreprise devrait nous alerter.

Par ailleurs, il a été noté que le processus entrepreneurial peut agir comme « support de résilience » pour les entrepreneurs (Bernard, 2008). Face à l'image d'un entrepreneur-inventeur génial, équilibré et positif, certaines études montrent que le choix de devenir entrepreneur fait souvent partie d'un processus de résilience au sens de Boris Cyrulnik, c'est-à-dire comme une opportunité de reconstruction de la personne après des épisodes difficiles. Chez des startuppers, une enquête sur une petite centaine d'entre eux en Midi- Pyrénées a montré que la création d'une start-up n'est pas en général le « résultat d'une prise de risque visionnaire d'une personne en situation stable, [mais] nettement plus fréquemment une solution parmi d'autres pour quelqu'un qui se trouve en situation instable, voire parfois le dos au mur » (Barthe, Chauvac et Grossetti, 2016). Notons toutefois que leur définition d'une start-up diffère sensiblement des profils orientés sur la netéconomie que nous avons interrogés dans cette étude ${ }^{6}$.

D'autres figures de l'anxiété peuvent s'appliquer aux start-uppers et aux entrepreneurs au sens large. Par exemple, les positions de leadership peuvent générer un mal-être important dans leur organisation naissante (Brasseur, 2012). De manière analogue, ils connaissent une situation de compétition permanente à la fois dans leur activité (face à des concurrents innovants ou face aux entreprises installées qu'ils espèrent détrôner), mais aussi pour l'accès aux ressources financières, humaines, logis-tiques, etc. Cette course permanente et cette remise en cause régulière rappellent par certains côtés l'anxiété compétitive décrite chez les sportifs dont les effets seraient ambivalents : "Pour certains, l'anxiété constitue un frein à la performance : l'athlète anxieux est un athlète fragi-lisé. D'autres la considèrent au contraire comme un moteur de l'action et citent en exemples ces athlètes qui ne sont performants que sous la pression de la compétition et l'état d'anxiété qu'elle engendre »(Debois, 2003).

Dans l'introduction d'un livre symptomatique de la littérature sur les start-up, Eric Ries (2011) écrit à propos de la start-up lean : " Ce mouvement cherche à faire en sorte que ceux d'entre nous qui désirent construire la prochaine grande chose $^{7}$ auront les outils dont nous avons besoin pour changer le monde. " II illustre la figure héroïque et média-tique du start-upper comme héros moderne, symbole à la fois de réus-site professionnelle, financière et sociale, se croyant capable d'inventer

${ }^{6}$ Dans cet article, les auteurs définissent la « start-up » sur le fait que « leurs fondateurs ont réussi à convaincre des organismes de soutien à l'innovation du caractère "innovant" de leur projet ».

${ }^{7}$ «The next Big Thing ». 
de nouveaux modes de fonctionnement aux activités qu'il concurrence (Uber, Airbnb, etc.). Nous savons maintenant qu'elle se double d'une réalité psychique plus incertaine et instable, que nous allons explorer.

\section{L'enquête : le numa...}

Le lieu de notre enquête est un incubateur et accélérateur ${ }^{8}$ de start-up emblématique de " l'écosystème " parisien des start- up créé à la suite de la bulle Internet des années 2000. La fondatrice, Marie-Vorgan Le Barzic, nous explique : " Je me suis attachée à redonner vie, redonner des moyens à ces entrepreneurs qui étaient encore vivants à la sortie de cette bulle. " Le numa se trouve rue du Caire, dans le $3^{\mathrm{e}}$ arrondissement de Paris. II s'agit d'un immeuble de six étages, très moderne, composée d'open spaces éclairés par de grandes fenêtres et des baies vitrées. Marie se sent investie d'une mission : « Ce qui m'habite et ce qui m'illu-mine, c'est de profiter du passage sur terre pour faire changer le monde dans un sens qui soit plus favorable à nos enfants. C'est ça le principe fondamental de mon action, depuis longtemps. " Une mission qui fait écho

à celle affichée sur le site web de l'incubateur : " numa soutient les entrepreneurs tech qui se donnent pour mission de répondre aux enjeux mondiaux de 2030. "Les 54 employés du numa accompagnent des centaines d'entrepreneurs chaque année à travers trois activités principales :

- Un espace de coworking: des postes avec wifi sont mis gratuitement à disposition des entrepreneurs, sur le principe du «premier arrivé, premier servi »;

- Un accélérateur de start-up : un programme très sélectif de quatre mois qui vise à accompagner des entreprises à fort potentiel à grand renfort de mentorat, coaching, formation et accès à des financements ;

- Une mission d'animation et de plaidoyer : le numa offre un riche programme événementiel pour promouvoir l'entrepreneuriat et faire grandir l'écosystème.

numa a par ailleurs récemment développé plusieurs antennes à l'étran-ger (Bangalore, Moscou, Casablanca, New York, Mexico).

De jeunes étudiants entrepreneurs peuvent librement utiliser l'espace de coworking du rez- de-chaussée alors que les étages supérieurs sont réservés à des start-up qui ont complété un processus de sélection extrême-ment strict (lors de la dernière promotion, cinq start -up ont été retenues sur 850 candidatures). Les rares heureux élus, qui ont su démontrer le potentiel de leur innovation et de leur business model, sont alors intégrés dans un programme d'accompagnement intensif de quatre mois, qui inclut du mentorat, des investissements et des mises en réseau (programme

${ }^{8}$ Accélérateur : un modèle d'incubateur à but lucratif, qui offre différents services d'accompagnement (mentorat, formation, etc.) en échange d'une part du capital. 
d'accélération). Ces « jeunes pousses » conjuguent une dimension numé-rique ou technologique avec un fort potentiel de croissance.

Dans les faits, peu de projets sont profitables et la plupart ne tiennent que par les capitaux investis en permanence, dans l'espoir qu'un grand groupe rachètera plus tard la « bonne idée » de la start-up, son image, ses algorithmes et sa communauté d'utilisateurs. Rappelons que de nombreuses entreprises du Net, y compris les plus importantes comme Amazon ou Twitter, n'ont pas été rentables pendant de très longues années, ce qui ne les a pas empêchées de connaître des succès considérables en Bourse. La situation des start-up, comme de leurs initiateurs, est donc financière-ment précaire, alors même que l'image extérieure de cet univers est celle des fortunes faites facilement par de jeunes et audacieux entrepreneurs.

\section{...et 19 start-uppers interviewés}

Les entretiens semi-directifs ont été menés auprès de fondateurs de start-up en cours " d'accélération ", d'anciennes lauréates du programme et des porteurs de projets plus récents. Leurs entreprises étaient arrivées

à des niveaux de maturité très différents puisqu'elles pouvaient avoir entre zéro et vingt-deux salariés et entre une et huit années d'existence. L'intégralité des

échanges a été filmée et a donné lieu à la réalisation d'un documentaire ${ }^{9}$. Les participants sont à l'image de la population rencontrée dans cet incubateur : jeunes (entre 21 et 40 ans), majoritairement de type caucasien et très souvent issus des grandes écoles (hec, Epitech, iae de Toulouse, Polytechnique, Sciences Po, Audencia, iep Aix-en-Provence, essec). En plus de ce bagage culturel élevé, ils bénéficient pour la plupart d'un capital économique familial important - puisqu'ils sont issus des classes socioprofessionnelles supérieures (fils de médecins, de chefs d'entreprise, d'enseignants, etc.). Nous avons une surreprésentation de femmes (cinq répondantes sur dix-huit) dans la mesure où elles ne repré-sentent qu'une très faible minorité des entrepreneurs qui fréquentent l'incubateur. Le matériau obtenu (vingt-cinq heures d'enregistrement et soixante-treize pages de verbatim sélectionnés) a été analysé dans une perspective socio-anthropologique.

\section{Des organisations en lutte pour leur survie}

Les premiers résultats de cette enquête montrent que l'anxiété est inhérente aux modalités de l'activité dans l'écosystème start-up. Elle s'explique d'abord par le contexte extrêmement compétitif dans lequel ces entrepreneurs évoluent. Le terme " accélérateur " n'est pas anodin, il s'agit avant tout d'aller plus vite que ses concurrents, d'anticiper les

${ }^{9}$ Changer le monde. Les entrepreneurs du numa, de Nathalie Luca et Romain Buquet, 2017. 
évolutions du marché et de rester à la pointe des évolutions techno-logiques. Antoine $^{10}$ résume simplement la situation : « En ce moment j'ai toujours du stress... on a un rythme très soutenu. " L'aspect commu-nautaire renforce aussi cette tension dans une forme d'émulation collec-tive. Cette concentration géographique de start-up dans cet incubateur avait été décryptée par Yan Dalla Pria et Jérôme Vicente comme source de " processus mimétiques [qui] constituent le ressort fondamental de la construction des formes d'action collective à l'œuvre dans le Silicon Sentier » (Dalla Pria et Vicente, 2006). Cette anxiété a été exprimée à diffé-rents degrés chez tous nos répondants.

Un autre élément partagé par l'ensemble des interviewés est la sensa-tion de vivre des pics émotionnels, positifs et négatifs : des pics d'eupho-rie extrême alternent avec des moments de grande angoisse. Ils sont nombreux à utiliser l'image des " montagnes russes émotionnelles ». Une entrepreneure expérimentée explique avec lucidité : « C'est un effet de levier : on monte hyper vite, mais la chute... on tombe de 120 mètres, on s'explose la gueule ! " (Laurie, 26 ans, diplômée de Sciences Po et hec). Ces pics émotionnels restent sous contrôle tant que l'entrepreneur est dans l'action, sans temps de réflexivité ou de recul, mais les moments de solitude, le soir ou le week-end, les rendent plus extrêmes encore : "Les week-ends c'est le moment où tu es seul, isolé, et ça fait très mal! Parce que tu as des "ups and downs" énormes: "on ne va jamais y arriver" ou alors au contraire on est hyper excité par ce qui s'est passé... beaucoup de remises en question parce que tu as du temps pour réfléchir. Ce sont des moments intenses, parfois vertigineux. II y a une euphorie énorme quand ça marche et il y a des vertiges énormes quand il y a des blocages " (Quentin, 33 ans, hec).

Les participants à notre étude disent ne pas connaître de "zone de confort ", car la viabilité économique de leur projet n'est pas assurée

à moyen terme et ils ne savent pas où ils en seront dans trois mois. Un sentiment à relativiser compte tenu du capital socio-économique dont ils jouissent par ailleurs. Cette anxiété peut être masquée par un espoir tenace, comme pour Antoine (24 ans, Epitech) : «Pour l'instant je n'en vis pas. J'en vivrai sûrement dans six mois. "Nous avons appris depuis que son entreprise a fait faillite. Un autre ne semble pas encore fixé sur son sort, alors qu'il consent des sacrifices importants depuis déjà deux ans : "Nous on est au stade de la pièce qui tourne sur le verre, qui devrait tomber dans le verre mais qui peut encore faire une petite surprise et tomber dehors " (David, 25 ans, iae de Toulouse). Une situation d'autant plus tendue qu'il partage une petite colocation avec ses deux associés et qu'aucun d'entre eux ne se rémunère sur le projet, comme beaucoup des entrepreneurs que nous avons interrogés. Alors que les plus jeunes ne bénéficient d'aucune protection sociale ni 
rémunération, ceux dont les start-up sont les plus matures se versent un salaire modeste - bien inférieur à celui qu'ils pourraient toucher comme salariés dans une entreprise. Enfin, ceux qui ont initié leur projet après quelques années de salariat bénéficient des allocations chômage $(60 \%$ du salaire précédent pendant vingt-quatre mois). Leur salut personnel se mélange alors avec celui de l'entreprise : " La peur c'est les besoins de financements. Tous les six mois se demander si l'entreprise va pouvoir survivre »(Georges, 28 ans, ingénieur).

Cette question de la survie de l'entreprise, non encore rentable et ne tenant que grâce aux apports permanents en capital d'investisseurs, est récurrente. Ainsi Laurie, du haut de ses 26 ans, nous raconte son histoire comme une épreuve surmontée mais éprouvante : “ $A ̀$ un moment on s'est retrouvés dans une situation où soit j'avais plusieurs millions d'euros sur le compte en banque, soit je virais les six personnes que j'avais choisies et que j'aime le plus sur terre, à savoir mes employés, qui ont des enfants...

Alors que j'avais 25 ans ! Eh bien voilà, c'est deux semaines où il faut s'inventer un estomac. Parce que ce n'est pas très rigolo... »

\section{Entre apothéose de soi et responsabilité d'autrui}

Les start-uppers s'identifient à leur projet. Ils savent qu'il y a $90 \%$ d'échec et travaillent si dur pour échapper à cette fatalité que, lorsque fina-lement ils échouent, beaucoup font une dépression. Laurie : " Je pense que ça fait des dégâts colossaux. J'ai des amis qui étaient entrepreneurs, qui ont fait faillite... ils ont tous fait une dépression, tous. Je pense qu'on met beaucoup trop de nous dans ce truc-là. C'est ça le problème de faire un bébé ! C'est pour ça que j'essaie de le décorréler de moi. » Plus ils ont conscience de la possibilité de l'échec, plus ils se donnent du mal pour l'éviter, et plus la chute est douloureuse, proportionnelle au niveau d'inves-tissement et, conséquemment, à l'épuisement dans lequel ils finissent l'aventure.

Avoir conscience du phénomène ne donne pas de clé pour lui échapper et la faillite éventuelle constitue toujours une blessure narcissique profonde du fait de l'investissement psychique et identitaire du start-upper dans son projet. II est très courant que le start-upper " mise tout " sur son entreprise, au niveau économique, affectif et psychique. Ainsi l'un d'entre eux a-t-il quitté son école de commerce en cours d'année pour se consacrer entiè-rement à son entreprise. II y consacre aussi le reliquat de son emprunt étudiant, au risque, en cas d'échec, de se retrouver sans diplôme et consi-dérablement endetté à titre personnel. $\mathrm{Ce}$ faisant, il ne fait que réitérer les exploits mythifiés de Steve Jobs (Apple) et de Mark Zuckerberg (Facebook), qui ont tous deux abandonné leurs universités avant d'être diplômés pour se consacrer totalement à leurs entreprises naissantes. En prenant ce risque, ils se mesurent donc, d'une façon elle aussi mimétique, à ceux qui sont les stars incontestées du domaine. 
Les tensions sont aussi accrues par des enjeux relationnels, notamment lorsque d'autres employés sont en jeu. Quand l'entrepreneur lève des fonds ou recrute une équipe, il étend sa responsabilité envers ceux qui lui ont fait confiance : les investisseurs, ses premiers employés et leurs familles éventuelles. Une responsabilité d'autant plus pesante qu'elle concerne des personnes qui ont cru en lui et adhéré à son projet. Pour Eddie (39 ans, Audencia), cela limite grandement la prétendue liberté dont jouiraient les créateurs d'entreprises : "Finalement je suis libre de faire ce que je veux, mais je dois le faire en tenant compte de tous ces collaborateurs qui ont confiance, qui me suivent. "Karen (22 ans, Sciences Po) précise : «Je pense qu'on a une certaine pression qui s'ajoute quand on lève un million. " Et quand Laurie se plaît à rêver d'un ailleurs, de nouvelles aventures et de repartir à zéro, ses responsabilités la rattrapent bien vite : «Après, on dit ça... C'est plus facile à dire quand tu as zéro employé que quand tu en as quinze, avec de l'argent sur le compte, des investisseurs à qui tu dois rendre des comptes... »

\section{Sisyphe ou Hippocrate au secours de Narcisse}

Une anecdote relatée dans un des entretiens lève le voile sur les coulisses psychologiques de ces entrepreneurs. Une start-uppeuse, accomplie et en apparence très confiante, raconte un dîner informel entre start-uppers au cours duquel la parole s'est libérée, loin des regards extérieurs et des exigences de performance. Tour à tour, les entrepreneurs présents ce soir-là ont partagé leurs souffrances intimes, jusqu'à reconnaître un usage régulier d'anxiolytiques. Elle réalise alors que les anxiétés vertigineuses qu'elle traverse les concernent finalement tous : «Pour la première fois je me suis dit, en fait, tout le monde le vit hyper mal, on fait tous semblant d'être des putains de rock stars et en fait... on est tous trop en galère ! Et ça, ça fait du bien ! Discuter de la solitude, des angoisses, etc. Litté- ralement, il y en avait la moitié sous calmants! »

À côté du choix « médical » de traiter par des produits ad hoc l'anxiété ressentie, l'autre stratégie de défense consiste en une fuite en avant dans le travail, en se «noyant dans l'action ». Sisyphe vient alors au secours de Narcisse : les entrepreneurs renouvellent sans cesse leur implication pour en apparence maximiser leurs chances de succès, mais en réalité conjurer leur anxiété, et ils y échouent en définitive toujours. Dès lors, ils développent une forme d'accoutumance, voire d'addiction, aux pics émotionnels vécus dans leur travail. Ce mécanisme de défense nous est dévoilé avec une grande spontanéité : « [Avec l'expérience] on devient à la fois un peu immunisé par rapport aux montagnes russes émotionnelles que procure l'entrepreneuriat et addict au fait que chaque journée il se passe quelque chose d'un peu incroyable. Je pense que je ne pourrai plus jamais remettre les pieds dans une boîte où je pourrais passer une journée sans qu'il ne se soit rien passé d'important » (Pierre, 32 ans, hec). 
L'anxiété et les souffrances initiales sont ainsi sublimées dans un engagement entrepreneurial renouvelé et permanent, pour s'accoutumer au quotidien dans un déséquilibre addictif.

\section{Fuir la grande entreprise et créer le bien-être au travail}

Les start-uppers que nous avons rencontrés livrent un discours extrê-mement critique face à la grande entreprise, ses processus, ses normes, son " aliénation ", vécue comme une forme d'emprisonnement, de renon-cement à sa liberté, à son potentiel créatif : « En étant salarié auparavant, je me suis toujours senti contraint, limité. Je ne me retrouvais pas dans les structures, les process " (Bernard, 44 ans, école de commerce). Cette critique revient comme un boomerang sur ceux qui connaissent des premiers succès, recrutent une équipe, se développent et craignent de devoir mettre en place la hiérarchie et les processus de contrôle qu'ils ont pourtant fuis :

"Ce qui me démotiverait ce serait de voir que ce que l'on fait n'a pas de sens. Moi-même recréer une organisation qui a ses propres process et revenir finalement dans ce que j'ai voulu quitter " (Georges, 28 ans, ingé- nieur). II y a ainsi une double peur : reproduire le même en cas de réussite ; retourner dans " la grande entreprise " pourtant honnie en cas d'échec. La situation, intenable dans tous les cas, est nécessairement anxiogène.

Ce rejet d'un ancien monde est-il pour autant original ? Dans les années 1990, Jean-Pierre Le Goff évoquait déjà des entrepreneurs d'un nouveau type, qui s'opposaient à l'ancienne économie, le modèle taylorien (Le Goff, 1992). Ces méthodes et discours de management reposaient sur le nécessaire embrigadement des cadres pour qu'ils adhèrent à la culture de l'entreprise, à grand renfort d'appel aux valeurs et de stimulation du sentiment d'appartenance. Le Goff montrait d'ailleurs que des méca-nismes similaires étaient déjà à l'œuvre dans le capitalisme paternaliste $\mathrm{du} \mathrm{xix}^{\mathrm{e}}$ siècle...

Les entretiens révèlent des pôles d'opposition en termes d'engagement, de force de rupture, de mode d'autorité qui donnent à penser la relation entre la start-up et la grande entreprise comme structurellement proche de celle, dialectique, à partir de laquelle Max Weber et Ernst Troeltsch travaillent la tension originelle entre secte et Église (Luca, 2004). Le premier élément de distinction est la conversion. Elle est opérationnelle ici dans le sens où les start-uppers vivent le salariat dans la grande entreprise comme relevant du parcours classique, subi, sans investissement $d u$ chercheur d'emploi. Pour la fondatrice du numa, il s'agit donc de « faire bouger les ordres établis ». Karen n'avait pas envie pour sa part de « mener une vie dans laquelle à la sortie des études je rentre dans une entreprise et je m'installe dans mon quotidien. [...] Parce que je n'ai pas envie de me faire à la médiocrité ». Créer une start-up, comme y travailler, demande de changer son rapport au travail, ce qui passe par la transformation de toute une série d'habitudes. Ainsi, l'un des start-uppers dira : "C'est une 
véritable conversion. " Pour Laurie, cela se manifeste notamment par la transformation vestimentaire et culinaire : elle est passée du tailleur au jean et a remplacé les sushis par une pizza/bière. Par ailleurs, elle ne " donne plus sa vie à quelqu'un d'autre que ça enrichissait », mais elle "crée des trucs » à elle. Être dans une start-up, c'est également être dans une demande d'adhésion à un projet, et par conséquent dans une quête de sens que l'on ne trouverait plus dans la grande entreprise : " II y a encore dix ou quinze ans, on pouvait se raconter que la grande entreprise était celle qui allait vous protéger. [...] la promesse de construction personnelle ne se fait plus au même endroit. [...] Le sens n'est plus à l'intérieur de ces grandes entreprises aujourd'hui. [...] Si j'ai 25 ans en 2016 en France, quels sont les moyens que j'ai pour me réaliser ? C'est assez simple : je vais créer mon entreprise. [...] Je pense que c'est beaucoup plus excitant pour un jeune diplômé de rejoindre une start-up » (Marie).

Le deuxième élément de tension est celui du mode d'autorité : face à la bureaucratie qui valorise une légitimité rationnelle-légale qui s'incarne dans la hiérarchie, le start-upper est un créateur d'un nouvel ordre, proche en cela du leader charismatique qui se doit de convaincre de la force, de l'originalité et de l'avenir de son projet auprès de ses potentiels financeurs, de ses collaborateurs, de ses stagiaires ou salariés. Chacun se retrouve pleinement engagé parce que pleinement convaincu, ce qui abolit toute nécessité de hiérarchie et rend secondaire la délimitation d'un temps de travail et l'obligation d'un salaire : la start- up regroupe des individus portés par une même volonté de donner vie et avenir au projet du leader-créateur. Ainsi, Laurie parle de ses salariés comme de "petites boules d'amour ». Christine (21 ans, école de commerce) ne peut pas comprendre qu'un stagiaire puisse ne pas répondre à ses e-mails le week-end ou quit-ter le travail à 17 heures - «Pour moi, week-end, soirée, journée, c'est la même chose » - et, en règle générale, ses stagiaires, bien que non indemnisés, adhèrent suffisamment au projet pour s'y donner sans compter. Ainsi, le niveau partagé d'investissement et de croyance dans les poten-tialités de la start-up semble pouvoir abolir toute forme hiérarchique au profit d'une atmosphère chaleureuse et familiale : « Le jour où j'ai des salariés je ne les considérerai pas comme des salariés mais comme une famille... c'est déjà le cas avec les stagiaires, même s'ils ne sont là que pour deux mois » (Christine).

Le troisième élément de tension est la force de rupture de la start-up : une volonté de rompre avec le fonctionnement des grandes entreprises vis-à-vis desquelles les start-uppers expriment leur détestation. La rupture se manifeste également dans "l'écosystème start-up » du fait même des très petites tailles des entreprises qui leur donne agilité et rapidité là où les grandes structures sont plus lentes et difficiles à réformer. Cela n'est pas sans conséquence sur ces dernières qui tentent désespérément de faire entrer « l'esprit start-up » dans leur management. 
Ainsi, le monde de la start-up, dans les discours des entrepreneurs, n'apparaît pas seulement comme « une assomption du sujet indépendant » (Giust Desprairies et Faure, 2015), mais également comme une commu-nauté soudée, efficace et solidaire face à l'image négative de la grande entreprise, lente, trop structurée, qui assujettit l'individu dans un travail dont il perd de vue la finalité. Cet imaginaire négatif de la grande entreprise se nourrit de l'image médiatique positive d'entrepreneurs ayant réussi dans l'informatique ou la netéconomie (Bezos, Zuckerberg, Musk, etc.), qui appa-raissent comme des « héros modernes » (McMinn, 2015). II y a certes une contradiction entre le refus de la " grande entreprise " et le désir de réussir comme d'autres entrepreneurs qui ont in fine créé de grandes entreprises, comme Amazon, Google ou Facebook, mais, pour les entrepreneurs de notre échantillon, ces exemples ne sont pas comparables aux entreprises classiques industrielles ou de service, ce sont des modèles positifs et désirables, car issus de start-up et ayant, d'une certaine façon et dans leur domaine, " changé le monde ».

\section{Le désir de changer le monde}

Luc Boltanski et Ève Chiapello différencient " trois esprits du capita-lisme ». Le premier lie destin de l'entreprise et destin familial, le deuxième repose sur la figure du directeur des grandes entreprises, le troisième est celui du capitalisme mondialisé. II se développe dans une situation de concurrence accrue et par le biais de nouvelles technologies. Les start-up relèvent de ce troisième esprit. Tout comme celles qui leur ont précédé, elles prétendent à leur tour " apaiser l'inquiétude que suscite trois questions :

- $\quad$ En quoi l'engagement dans les processus d'accumulation capi-taliste estil source d'enthousiasme, y compris pour ceux qui ne seront pas nécessairement les premiers bénéficiaires des profits réalisés?

- $\quad$ Dans quelle mesure ceux qui s'impliquent dans le cosmos capita-liste peuvent-ils être assurés d'une sécurité minimale pour eux et pour leurs enfants ?

- Comment justifier en termes de bien commun la participation à l'entreprise capitaliste et défendre, face aux accusations d'injustice, la façon dont elle est animée et gérée ? 》 (Boltanski et Chiapello, 1999, p. 53).

Le désir de réussir des start-uppers n'est pas prioritairement ou unique-ment motivé par des considérations économiques, même si elles sont bien évidemment importantes. Leur volonté de prouver leur valeur personnelle et, plus encore, de réaliser quelque chose qui les dépasse et qui trans-forme à un certain niveau leur société, voire le monde, est un puissant moteur. En cela, les start- uppers sont tournés vers un idéal, non pas un idéal abstrait, si élevé ou éloigné qu'aucun moyen ne pourrait être mis au service de sa réalisation, mais au contraire un idéal à portée d'action, 
telle que John Dewey le définit, dont ils peuvent faire l'expérience parce qu'il se concrétise dans chaque situation (Dewey et Tufts, 1908). Créer une startup, ce n'est pas seulement donner du sens à sa vie profession-nelle, c'est aussi, pour beaucoup, réparer les imperfections de la société, trouver de nouveaux business model capables de définir de nouvelles normes. Ce n'est pas seulement se sauver, c'est aussi sauver le monde par touches successives et en fonction de son domaine de compétence : «À un moment je me suis dit : est-ce que je veux être avocat dans vingt ans,

à un moment où tout change ? Non ! C'est le moment où, au contraire, il faut faire partie de ceux qui vont changer le monde » (Quentin).

L'engagement entrepreneurial connaît un essor sans précédent parmi les jeunes générations, au moins aussi puissant que le désinvestissement collectif de la sphère politique. Après les grands mouvements politiques du $\mathrm{xx}^{\mathrm{e}}$ siècle, la start-up apparaît pour certains comme un moyen plus sûr d'imprimer sa marque sur le monde (Roche, 2003). Réenchanter le monde par l'entrepreneuriat revient à combler les vides laissés par le reflux du religieux puis du politique, comme l'explique la fondatrice de l'incubateur : " Quand on ne croit plus en Dieu, qu'on ne croit plus au président, qu'on ne croit plus aux institutions, qu'est ce qui reste ? Eh bien, la possibilité de reconstruire quelque chose. "Après l'engagement politique collectif, un engagement entrepreneurial communautaire ? La question mérite d'autant plus d'être posée que nombre de start-uppers sont les héritiers de ceux qui animaient les mouvements de la contre-culture des années 1970, à l'image de San Francisco, qui fut un pôle du mouvement hippie dans les années 1960 et est aujourd'hui la capitale incontestée des start-up technologiques. L'engouement pour la création de sa propre entreprise apparaît ainsi comme un avatar des désirs révolutionnaires et politiques des années 1960/1980, dans une déclinaison plus économique et entrepreneuriale, cohérente avec le mythe fondateur américain du self-made-man : "On convoque régulièrement la collaboration, le partage, la dématérialisation, l'horizontalité, les réseaux, la fin du "top-down", sans oublier l'éternelle révolution qui est presque devenue un préfixe. Ce lexique n'aurait pas déplu à un Bakounine, et pour cause, il provient tout droit de la gauche radicale américaine, massivement concentrée en Californie et dans la Vallée pendant les années 1970. [...] Au milieu des années 1990, avec la libéralisation d'Internet, cet héritage s'est cristallisé sous la bannière de la Nouvelle Économie » (VionDury, 2016, p. 207).

\section{Conclusion : l'anxiété créatrice des héros « aux pieds d'argile »}

Le désir d'échapper au modèle honni de la grande entreprise est un des moteurs de l'engagement entrepreneurial, qui se double souvent d'une sorte d'anxiété plus globale face aux évolutions du monde moderne. Mais cette anxiété est créatrice : l'entrepreneur apparaît comme un " thauma-turge » qui veut changer le monde, au moins au niveau de son domaine 
d'activités, en offrant des services sous des formes nouvelles ou en réparant des situations vécues comme des "injustices » dans l'offre de services préexistants. Là où d'autres figures salvatrices apparaissaient dans le passé comme celles du leader politique ou de l'intellectuel engagé, le sentiment domine que, pour ces entrepreneurs, c'est par de nouvelles entreprises comme la leur que les problèmes du monde pourront être résolus. Ce rejet de la grande entreprise et cet engagement au travers des start-up ne peut s'analyser en dehors du contexte économique et social dans lequel ils se produisent, ils font écho à ce " réenchantement de l'entreprise " qu'évoquait Jean Bunel il y a trente ans, qu'il analysait comme

« un effet inattendu de la crise économique et du chômage » (Bunel, 1986, p. 259).

Numa ne définit pas vraiment un groupe au sens classique et formel du terme, ne serait-ce que parce que chacun a une activité singulière, un projet personnel qui le distingue et le sépare des autres. Pour autant, on retrouve ici les éléments de construction d'une identité collective par comportement mimétique (Dalla Pria et Vicente, 2006), renforcé par l'existence même de l'incubateur, la mise en commun de pratiques colla-boratives et la compétition permanente (pour des prix, des financements) auprès d'investisseurs - situation de « mise en commun » de ressources, de sentiments et d'expériences qui peuvent auto-entretenir les mécanismes d'anxiété. Bien que ce " groupe " d'entrepreneurs n'en soit pas vraiment un, on a le sentiment de rencontrer ici, par la similitude des propos, par l'homogénéité de l'imaginaire mobilisé, ce qu'évoque René Kaës quand il parle d'" alliance inconsciente " (Arnaud, 2004). Plus encore, le refus de la grande entreprise honnie, refus partagé par tous de façon plus ou moins explicite et ancré dans des expériences diverses mais convergentes, fait penser à une sorte de " stratégie de désenlisement " (Jullien, 2016) face à un malaise persistant, voire comme une tentative d'échapper à la dépres-sion. Le modèle de la start-up définit une forme contemporaine d'« imagi-naire leurrant » (Enriquez, 1990) qui se trouve médiatisé et renforcé par quelques grandes figures emblématiques et, in fine, les conditions initiales du malaise ressenti, ainsi que les faibles chances de succès associées, font que cet imaginaire est très vite confronté à la faillite du projet et à son corrélat, la défaillance du sujet. L'entrepreneur apparaît ainsi comme un héros anxieux, un prophète incertain, un « appelé » qui doute.

\section{Bibliographie}

Abdelsamad, M.H. ; Kindling, A.T. 1978. "Why small businesses fail ", sam Advanced Management Journal, 43, 2, 24-32.

Arnaud, G. 2004. Psychanalyse et organisations, Paris, Armand Colin.

Auroux, S. 1990. "Le dictionnaire des notions philosophiques» », Stanford French Review, $14,3,105-138$. 
Barthe, J.-F. ; Chauvac, N. ; Grossetti, M. 2016. « Entrepreneurs de circonstance : une enquête sociologique sur les fondateurs de start-up en France ", Revue de l'entrepreneuriat, 15, 3, 163-180.

Barus-Michel, J. ; Enriquez, E. ; Lévy, A. (sous la direction de). 2002. Vocabulaire de psychosociologie, Toulouse, érès.

Bernard, M.-J. 2008. «L'entrepreneuriat comme un processus de résilience », Revue internationale de psychosociologie, 14, 32, 119-140.

Blanchflower, D.G. ; Oswald, A.J. 1998. «What Makes an Entrepreneur? », Journal of Labor Economics, 16, 1, 26-60.

Boltanski, L. ; Chiapello, Ė. 1999. Le nouvel esprit du capitalisme, Paris, Gallimard. Boudon, R. ; Bourricaud, F. 1990. «Valeurs », dans Dictionnaire critique de la sociologie, Paris, Puf.

Bouilloud, J.-P. 2012. Entre l'enclume et le marteau : les cadres pris au piège, Paris, Le Seuil.

Brandstätter, H. 1997. « Becoming an entrepreneur. A question of personality structure? », Journal of Economic Psychology, 18, 2-3, 157-177.

Brasseur, M. 2012. "Être chef malgré soi », Revue internationale de psychosociologie et de gestion des comportements organisationnels, 18, 45, 151-160.

Bunel, J. 1986. "Le réenchantement de l'entreprise ", Sociologie du travail, 28, 3, 251-264.

Busenitz, L.W. ; Barney, J.B. 1997. "Differences between entrepreneurs and managers in large organizations: Biases and heuristics in strategic decision-making ", Journal of Business Venturing, 12, 1, 9-30.

Chiapello, È. 1998. Artistes versus managers: le management culturel face à la critique artiste, Paris, Métailié.

Chiapello, È. 2004. "La contestation du capitalisme et I'histoire du management », Les échos, 25 novembre.

Clot, Y. ; Lhuilier, D. 2010. Travail et santé : ouvertures cliniques, Toulouse, érès.

Cross, B. ; Travaglione, A. 2003. "The untold story: is the entrepreneur of the $21^{\text {st }}$ century defined by emotional intelligence? ", The International Journal of Organizational Analysis, 11, 3, 221-228.

Dalla Pria, Y. ; Vicente, J. 2006. "Processus mimétiques et identité collective : gloire et déclin du "Silicon Sentier" ", Revue française de sociologie, 47, 2, 293-317.

Debois, N. 2003. «De l'anxiété aux émotions compétitives : état de la recherche sur les états affectifs en psychologie du sport ", Staps, 62, 3, 21-42.

Dewey, J. ; Tufts, J.H. 1908 « The virtues », dans Ethics, New York, Henry Holt and Company, 399-424.

Dimov, D. 2010. « Nascent Entrepreneurs and Venture Emergence: Opportunity Confidence, Human Capital and Early Planning ", Journal of Management Studies, 47, 6, 1123-1153.

Dujarier, M.-A. 2006. L'idéal au travail, Paris, Puf.

Enriquez, E. 1990. "L'entreprise comme lien social : un colosse aux pieds d'argile », Recherches et prévisions, 24, 1.

Fraser, S. ; Greene, F.J. 2006. « The effects of experience on entrepreneurial optimism and uncertainty », Economica, 73, 290, 169-192.

Freeman, M.A. ; Johnson, S.L. ; Staudenmaier, P.J. ; Zisser, M.R. 2015. « Are Entrepreneurs "Touched with Fire"? », [en ligne] http://www.michaelafreemanmd. 

pub\%20n)\%204-17-15.pdf .

Freud, S. 1975. Inhibition, symptôme et angoisse, Paris, Puf, 51-56.

Gartner, W.B. 2004. Handbook of Entrepreneurial Dynamics: The Process of Business Creation, Londres, SAGE.

Giust-Desprairies, F. ; Faure, C. 2015. Figures de l'imaginaire contemporain, Paris, Éditions des archives contemporaines.

Gomez-Breysse, M. 2016. "L'entrepreneur "lifestyle" », Revue de l'entrepreneuriat, 15, 3, 231-256.

Hanique, F. 2014. Le sens du travail, Toulouse, érès.

Hmieleski, K.M. ; Baron, R.A. 2009. " Entrepreneurs' Optimism and New Venture Performance: A Social Cognitive Perspective », Academy of Management Journal, 52, 3, 473-488.

Jullien, F. 2016. Vivre en existant : Une nouvelle éthique, Paris, Gallimard.

Laplanche, J. ; Pontalis, J.-B. 1968. Vocabulaire de la psychanalyse, Paris, Puf. Le Goff, J.P. 1992. Le mythe de l'entreprise : critique de l'idéologie managériale,

Paris, La Découverte.

Linhart, D. ; Rist, B. ; Durand, E. 2002. Perte d'emploi, perte de soi, Paris, érès. Low, M.B. ; MacMillan, I.C. 1988. " Entrepreneurship: Past research and future

challenges ", Journal of management, 14, 2, 139-161.

Lowe, R.A. ; Ziedonis, A.A. 2006. « Overoptimism and the performance of entre-preneurial firms », Management Science, 52, 2, 173-186.

Luca, N. 2004. Les sectes, Paris, Puf, 2016.

McMinn, S. 2015, "Entrepreneurs are our modern heroes », DecodeDC, [en ligne] http://www.decodedc.com/entrepreneurs-modern-heroes/

Moules, J. 2015. « Start-up costs for MBA graduates pay off », Financial Times, 18 janvier.

Pérezts, M. ; Bouilloud, J.-P. ; Gaulejac (de), V. 2011. "Serving Two Masters: The Contradictory Organization as an Ethical Challenge for Managerial Responsibility », Journal of Business Ethics, 101, 33-44.

Rauch, A. ; Frese, M. 2007. "Let's put the person back into entrepreneurship research: A meta-analysis on the relationship between business owners' personality traits, business creation, and success ", European Journal of Work and Organizational Psychology, 16, 4, 353-385.

Ries, E. 2011. The lean startup: How today's entrepreneurs use continuous inno-vation to create radically successful businesses, New York, Crown Books.

Roche, L. 2003. “ La start-up est "volonté de puissance" », Market Management, 3, 1, 5664.

Schumpeter, J.A. 1934. The Theory of Economic Development: An Inquiry Into Profits, Capital, Credit, Interest, and the Business Cycle, New Brunswick, Transaction Publishers.

Sexton, D.L. ; Bowman, N. 1985. "The entrepreneur: A capable executive and more », Journal of Business Venturing, 1, 1, 129-140.

Singh, S. 1989. "Personality Characteristics, Work Values, and Life Styles of Fast- and Slow-Progressing Small-Scale Industrial Entrepreneurs ", The Journal of Social Psychology, 129, 6, 801-805.

Vion-Dury, P. 2016. La nouvelle servitude volontaire : enquête sur le projet politique de la Silicon Valley, Limoges, Fyp éditions. 
Romain Buquet, Nathalie Luca et Jean-Philippe Bouilloud,

Malaise dans les start-up. Entre désir héroïque et anxiété créatrice

\section{Résumé}

Cet article porte sur l'anxiété des entrepreneurs du numérique, ou start-uppers, souvent présentés comme des héros modernes. Dix-neuf entretiens cliniques de type " récit de vie » ont été conduits par une anthropologue et un chercheur en gestion dans un incubateur phare de start-up parisiennes : le numa. Ces résultats exploratoires n'ont pas volonté à être généralisés et demanderaient d'être approfondis par d'autres études complémentaires. Ils montrent que ces jeunes entrepreneurs, réunis par un capital culturel et économique fort et un rejet paradoxal de la grande entreprise, construisent une identité collective par com- portement mimétique marqué par une ambivalence entre peur de l'échec et investissement narcissique majeur dans leur projet. Ils sont soumis à une anxiété créatrice qui lie leur engagement et leur volonté de changer le monde.

\section{Mots-clés}

Start-up, anxiété, numa, engagement, récits de vie, entrepreneurs.

Romain Buquet, Nathalie Luca et Jean-Philippe Bouilloud, Malaise in start-ups. Between heroic desire and creative anxiety

\section{Abstract}

This paper focuses on the anxiety of digital entrepreneurs, or start-uppers, often portrayed as modern heroes. Nineteen clinical "life story" interviews were conducted by an anthropologist and a management researcher in a leading Parisian start-up incubator: numa. These exploratory results cannot be generalized and would require further research. They show that these young entrepreneurs, bound together by a strong cultural and economic capital and a paradoxical rejection of big corporations, build a collective identity by mimetic behavior marked by ambivalence between fear of failure and a major narcissistic investment in their project. They are subject to a creative anxiety that binds their commitment and willingness to change the world.

\section{Keywords}

Start-up, anxiety, numa, commitment, life stories, entrepreneurs. 